\title{
Theoretical analysis on the extension of a piezoelectric semi-conductor nanowire: Effects of flexoelectricity and strain gradient
}

Cite as: J. Appl. Phys. 127, 085707 (2020); https://doi.org/10.1063/1.5131388

Submitted: 12 October 2019 . Accepted: 19 February 2020 . Published Online: 28 February 2020

MingHao Zhao, Xiang Liu, CuiYing Fan, Chunsheng Lu (D), and BingBing Wang (D)

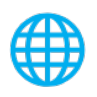

Lock-in Amplifiers Find out more today
Zurich

Instruments

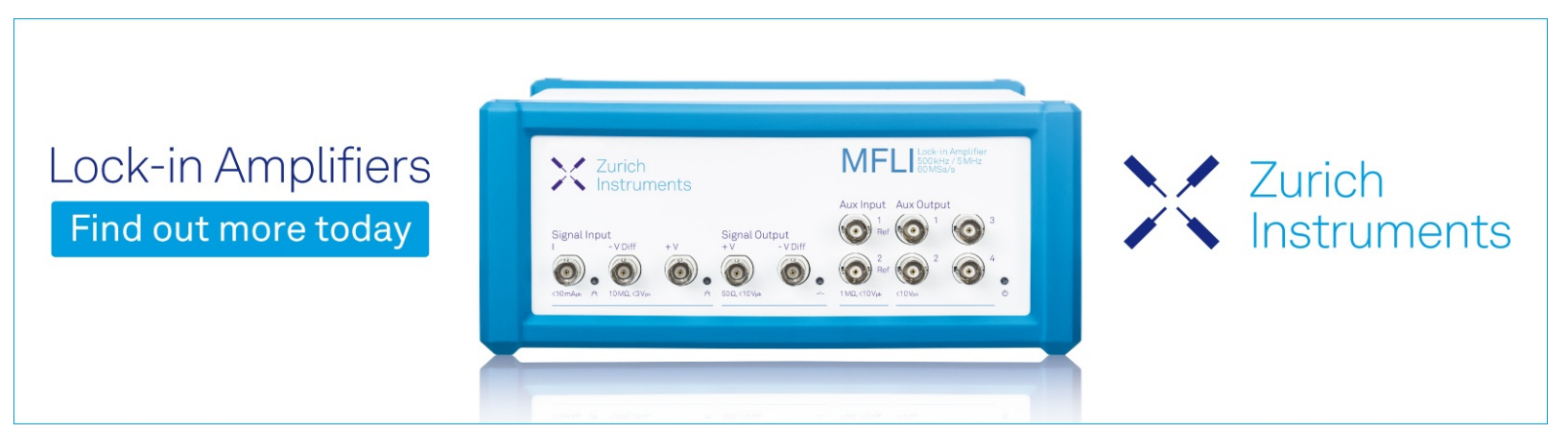




\title{
Theoretical analysis on the extension of a piezoelectric semi-conductor nanowire: Effects of flexoelectricity and strain gradient
}

Cite as: J. Appl. Phys. 127, 085707 (2020); doi: 10.1063/1.5131388

Submitted: 12 October 2019 . Accepted: 19 February 2020 .

Published Online: 28 February 2020

MingHao Zhao, ${ }^{1,2}$ Xiang Liu, ${ }^{7}$ CuiYing Fan, ${ }^{7}$ Chunsheng Lu, ${ }^{3}$ (D) and BingBing Wang ${ }^{1, a)}$ (D)

\begin{abstract}
AFFILIATIONS
${ }^{1}$ School of Mechanics and Safety Engineering, Zhengzhou University, Zhengzhou, Henan 450001, China

${ }^{2}$ Henan Key Engineering Laboratory for Anti-Fatigue Manufacturing Technology and School of Mechanical Engineering, Zhengzhou University, Zhengzhou, Henan 450001, China

${ }^{3}$ School of Civil and Mechanical Engineering, Curtin University, Perth, Western Australia 6845, Australia
\end{abstract}

${ }^{a)}$ Author to whom correspondence should be addressed: bbwang@zzu.edu.ch

\begin{abstract}
One-dimensional piezoelectric semiconductor (PSC) nanowires have been widely used in smart structures and devices; however, few theoretical studies on their nano-size effects have been carried out. In this paper, a theoretical analysis of one-dimensional $n$-type PSC nanowires under an axial force was carried out, with consideration of the flexoelectric and strain gradient effects. Exact solutions were obtained based on the differential operator theory. The flexoelectric and strain gradient effects on the internal electromechanical field and carrier concentration have been discussed. It can be shown that the flexoelectric effect weakens the piezoelectricity of a PSC nanowire while the strain gradient has the opposite effect, with an amplitude enhancement that is dependent on the flexoelectric and inner scale coefficients.
\end{abstract}

Published under license by AIP Publishing. https://doi.org/10.1063/1.5131388

\section{INTRODUCTION}

Piezoelectric semiconductors (PSCs) have been widely applied in intelligent structures and electromechanical devices ${ }^{1-4}$ because of their piezoelectric and semi-conductive properties. $^{5-7}$ A key characteristic of PSCs is the interaction between the internal electric field and carriers under an applied bias voltage or mechanical force. This makes PSCs exhibit novel mechanical, electronic, and optical behavior. ${ }^{8,9}$ Recently, various one-dimensional (1D) PSC structures (e.g., nanowires, nanobelts, and nanotubes) have been fabricated, ${ }^{10-12}$ and have been applied for nano-generators that convert mechanical energy into electrical energy, ${ }^{13-15}$ such as piezoelectric diodes ${ }^{16}$ and piezoelectric sensors. ${ }^{17}$

For PSC applications, both the piezoelectric and semiconductor effects must be taken into account. ${ }^{18}$ Because of their special features and excellent performance, significant research efforts have been devoted to PSC nanowires. For example, using a perturbation method, Gao and Wang ${ }^{19}$ deduced a potential analysis formula for a PSC nanowire under a lateral force without consideration of the deformation effects of an electric field, that is, their model was semi-coupled. Additionally, a fully coupled PSC nanowire subjected to a lateral force was studied using the finite element method. ${ }^{20,21}$ Zhang et al. ${ }^{22}$ established a continuous model of a 1D PSC, and then calculated the internal electro-mechanic field and carrier concentration along the nanowire under tension. Recently, the effect of an end force on charge carrier motion in a 1D PSC structure was investigated under extension. $^{23-27}$

When the structure of a material is reduced to micrometer or sub-micrometer dimensions, it begins to exhibit significant size effects. ${ }^{28-30}$ The strain gradient model proposed by Aifantis et al., ${ }^{31,32}$ with only one inner scale parameter, can succinctly describe the size effects in a material. For dielectric materials, the flexoelectric effect ${ }^{33-35}$ should also be considered, especially at nano-scales. ${ }^{36-39}$ The flexoelectric effect mainly reflects the relationship between the strain gradient and polarization. ${ }^{40}$ Shen et al. ${ }^{41,42}$ studied the potential distribution of $\mathrm{ZnO}$ nanowires and found that the flexoelectric effect has a large influence on the potential; however, they did not take the material's semi-conductive properties into account. Wang and Wang ${ }^{43}$ investigated the potential distribution of 
PSC structures, with consideration of the flexoelectric effect of loworder stresses but without the strain gradient. Because both the strain gradient and flexoelectricity may significantly affect the behavior of PSC nanowires, these two effects should simultaneously be introduced when studying their influence on the internal electromechanical field and carrier concentration.

This paper is organized as follows: the basic equations and general boundary conditions of a 1D PSC are described in Sec. II; the expressions of the electromechanical field and carriers along a nanowire are given in Sec. III; the influence of strain gradient and flexoelectric effects on the electromechanical field and carriers is discussed in Sec. IV; and finally, the main conclusions are presented in Sec. V.

\section{BASIC EQUATIONS AND BOUNDARY CONDITIONS}

First, let us consider an $n$-type PSC nanowire with a length $2 L$ and the cross-sectional area $A$, as illustrated in Fig. 1. Because the nanowire length is much larger than the characteristic size of its cross section, a 1D model can be introduced. For simplification, the influence of a physical force term and carrier recombination and generation are ignored.

According to the force balance principle with a strain gradient effect, the electrostatic Gauss theorem with the flexoelectric effect, and the principle of charge conservation, the governing equations for a $1 \mathrm{D}$ PSC $^{8,41,44}$ can be represented as

$$
\begin{gathered}
\frac{d \sigma_{z}}{d z}-\frac{d^{2} \tau_{z}}{d z^{2}}=0, \\
\frac{d D_{z}}{d \mathrm{z}}=q\left(-n+N_{D}^{+}\right), \\
\frac{d J_{\mathrm{z}}}{d z}=0,
\end{gathered}
$$

where $\sigma_{z}, \tau_{z}, D_{z}$, and $J_{z}$ are the stress, the higher-order stress term (with dimensions of $[\mathrm{F}][\mathrm{L}]^{-1}$ ), electrical displacement, and electron current density, respectively. $\mathrm{N}_{D}^{+}$is the impurity concentration of the donors, $n$ is the concentration of electrons, and $q$ is the absolute value of charge carried by a single electron.

In classical (local) continuum-based theories, the stress at one point only depends on the strain, while in the strain gradient theory (a type of non-local theory), the equivalent (or non-local) stress $\hat{\sigma}_{z}=\sigma_{z}-\frac{d \tau_{z}}{d z}$ depends on both the strain and its gradient, which is called the strain gradient effect. In the strain gradient theory, the

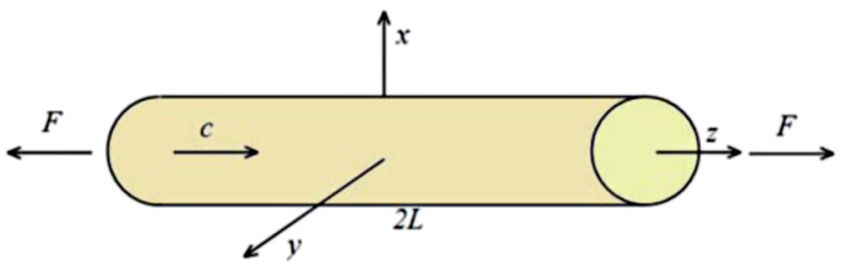

FIG. 1. Schematic illustration of a PSC nanowire under an axial force, where the $c$-axis is along the $z$ direction. strain gradient contributes to the higher-order stress by the term $c_{33} g^{2} \frac{d^{2} u}{d z^{2}}$ in a $1 \mathrm{D}$ case, where $c_{33}$ is the elastic modulus and $g$ is the non-local coefficient (or inner scale coefficient) that represents a material length scale. Flexoelectricity implies that strain gradients can induce polarization, which is

$$
P_{z}=f_{3} \frac{d^{2} u}{d z^{2}}
$$

where $f_{3}$ is the flexoelectric coefficient. With consideration of the strain gradient and the flexoelectricity, the 1D PSC constitutive equation $^{8,41,44}$ can be written as

$$
\begin{gathered}
\sigma_{z}=c_{33} \frac{d u}{d z}+e_{33} \frac{d \varphi}{d z}, \\
\tau_{z}=c_{33} g^{2} \frac{d^{2} u}{d z^{2}}+f_{3} \frac{d \varphi}{d z}, \\
D_{z}=e_{33} \frac{d u}{d z}-k_{33} \frac{d \varphi}{d z}+P_{z}, \\
J_{z}=-q n \mu_{33}^{n} \frac{d \varphi}{d z}+q d_{33}^{n} \frac{d \Delta n}{d z},
\end{gathered}
$$

where $u$ is the displacement of a PSC along the $z$-axis direction in the Cartesian coordinate system; $\varphi$ is the internal electric potential; $e_{33}$ and $k_{33}$ represent piezoelectric and dielectric coefficients, respectively; and $\mu_{33}^{n}$ and $d_{33}^{n}$ are the mobility and diffusion coefficients of electrons, respectively. It should be noted that the second term on the right side of Eq. (3b) denotes the contribution of the flexoelectric coefficient to the higher-order stress.

Without an applied external load, we have

$$
n_{0}=N_{D}^{+},
$$

and after loading, we obtain

$$
n=n_{0}+\Delta n,
$$

where $\Delta n$ is the perturbation of the carrier concentration.

In addition, the nanowire is assumed to be electrically neutral for the reference state. Therefore, $\Delta n$ must satisfy the global chargeneutral conditions, namely,

$$
\int_{-L}^{L} \Delta n d z=0
$$

Then, Eq. (1b) becomes

$$
\frac{d D_{z}}{d z}=-q \Delta n
$$

In the case of a small $\Delta n$, Eq. (3d) can be linearized ${ }^{22}$ as

$$
J_{z}=-q n_{0} \mu_{33}^{n} \frac{d \varphi}{d z}+q d_{33}^{n} \frac{d \Delta n}{d z} .
$$


For a 1D PSC, the general boundary conditions are

$$
\begin{gathered}
u=\bar{u}, \quad \hat{\sigma}=\sigma_{z}-\frac{d \tau_{z}}{d z}=\bar{\sigma}, \\
\frac{d u}{d z}=\bar{\gamma}, \quad \tau_{z}=\bar{\tau}, \\
\varphi=\bar{\varphi}, \quad D_{\mathrm{z}}=\bar{D} \\
\Delta n=\overline{\Delta n}, \quad J_{\mathrm{z}}=\bar{J},
\end{gathered}
$$

where $\bar{u}, \bar{\gamma}, \bar{\varphi}$, and $\overline{\Delta n}$ are the fixed displacement, displacement gradient, electric potential, and carrier concentration change, respectively, and $\bar{\sigma}, \bar{\tau}, \bar{D}$, and $\bar{J}$ are the applied stress, higher-order stress, electric displacement, and electric current, respectively.

\section{THE NANOWIRE UNDER AN AXIAL FORCE}

As illustrated in Fig. 1, the nanowire boundary is taken as electrical isolation. Hence, there is no current inflow (or outflow) at the two ends, and only the stress boundary conditions are necessary, that is

$$
\begin{gathered}
\hat{\sigma}( \pm L)=\frac{F}{A} \\
\tau_{z}( \pm L)=0 \\
D_{z}( \pm L)=0, \\
J_{z}( \pm L)=0 .
\end{gathered}
$$

According to Eq. (1c), $J_{z}$ is a constant and $J_{z}=0$. In combination with the current boundary condition in Eq. (10), Eq. (8) can be rewritten as

$$
-q n_{0} \mu_{33} \frac{d \varphi}{d z}+q d_{33} \frac{d \Delta n}{d z}=0
$$

Integrating Eq. (11) gives

$$
-q n_{0} \mu_{33} \varphi+q d_{33} \Delta n=C,
$$

and then we obtain

$$
\Delta n=\frac{\mu_{33}}{d_{33}} n_{0} \varphi+\frac{C}{q d_{33}},
$$

where $C$ is an integral constant. Taking the zero potential at the middle of the nanowire into account [i.e., $\varphi(0)=0$ and $u(0)=0$ ], we have $\Delta n(0)=0$ and thus $C=0$. Here, it is worth noting that $u(0)=0$ is introduced to eliminate the rigid displacement, and if necessary, any point can be fixed on the nanowire as the zero displacement point.

Substituting Eq. (3) into Eq. (1) leads to

$$
\begin{aligned}
& \frac{c_{33} d^{2} u}{d z^{2}}-c_{33} g^{2} \frac{d^{4} u}{d z^{4}}+e_{33} \frac{d^{2} \varphi}{d z^{2}}-f_{3} \frac{d^{3} \varphi}{d z^{3}}=0, \\
& e_{33} \frac{d^{2} u}{d z^{2}}+f_{3} \frac{d^{3} u}{d z^{3}}-k_{33} \frac{d^{2} \varphi}{d z^{2}}+q \frac{\mu_{33}}{d_{33}} n_{0} \varphi=0 .
\end{aligned}
$$

Further, by defining $U=\frac{d^{2} u}{d z^{2}}$, we have

$$
\begin{gathered}
c_{33} U-c_{33} g^{2} \frac{d^{2} U}{d z^{2}}+e_{33} \frac{d^{2} \varphi}{d z^{2}}-f_{3} \frac{d^{3} \varphi}{d z^{3}}=0, \\
e_{33} U+f_{3} \frac{d U}{d z}-k_{33} \frac{d^{2} \varphi}{d z^{2}}+q \frac{\mu_{33}}{d_{33}} n_{0} \varphi=0,
\end{gathered}
$$

and then its matrix form is

$$
\left[\begin{array}{cc}
c_{33}-c_{33} g^{2} \frac{d^{2}}{d z^{2}} & e_{33} \frac{d^{2}}{d z^{2}}-f_{3} \frac{d^{3}}{d z^{3}} \\
e_{33}+f_{3} \frac{d}{d z} & -k_{33} \frac{d^{2}}{d z^{2}}+q \frac{\mu_{33}}{d_{33}} n_{0}
\end{array}\right]\left[\begin{array}{l}
U \\
\varphi
\end{array}\right]=\left[\begin{array}{l}
0 \\
0
\end{array}\right] .
$$

According to the differential operator theory, Eq. (16) can be represented as

$$
\left[\begin{array}{cc}
c_{33}-c_{33} g^{2} D^{2} & e_{33} D^{2}-f_{3} D^{3} \\
e_{33}+f_{3} D & -k_{33} D^{2}+q \frac{\mu_{33}}{d_{33}} n_{0}
\end{array}\right]\left[\begin{array}{l}
U \\
\varphi
\end{array}\right]=\left[\begin{array}{l}
0 \\
0
\end{array}\right]
$$

with the notations $\frac{d}{d z}=D, \frac{d^{2}}{d z^{2}}=D^{2}, \frac{d^{3}}{d z^{3}}=D^{3}$, and thus, its characteristic equation is

$$
\alpha_{1} D^{4}+\alpha_{2} D^{2}+\alpha_{3}=0
$$

where

$$
\begin{gathered}
\alpha_{1}=c_{33} k_{33} g^{2}+f_{3}^{2}, \\
\alpha_{2}=-\left(c_{33} k_{33}+c_{33} g^{2} q \frac{\mu_{33}}{d_{33}} n_{0}+e_{33}^{2}\right), \\
\alpha_{3}=c_{33} q \frac{\mu_{33}}{d_{33}} n_{0} .
\end{gathered}
$$

Solving Eq. (18) gives the four real eigenvalues,

$$
\begin{aligned}
& r_{1}=-r_{2}=\sqrt{\frac{-\frac{\alpha_{2}}{\alpha_{1}}+\frac{\sqrt{\alpha_{2}^{2}-4 \alpha_{1} \alpha_{3}}}{\alpha_{1}}}{2}}, \\
& r_{3}=-r_{4}=\sqrt{\frac{-\frac{\alpha_{2}}{\alpha_{1}}-\frac{\sqrt{\alpha_{2}^{2}-4 \alpha_{1} \alpha_{3}}}{\alpha_{1}}}{2}} .
\end{aligned}
$$

Based on the differential operator theory, $\varphi$ and $U$ can be expressed as

$\varphi=C_{1} \cosh r_{1} x+C_{2} \sinh r_{1} x+C_{3} \cosh r_{3} x+C_{4} \sinh r_{3} x$,

$U=K_{1} \cosh r_{1} x+K_{2} \sinh r_{1} x+K_{3} \cosh r_{3} x+K_{4} \sinh r_{3} x$,

where the parameters $\left(C_{1}-C_{4}\right.$ and $\left.K_{1}-K_{4}\right)$ are constants. 
(a)

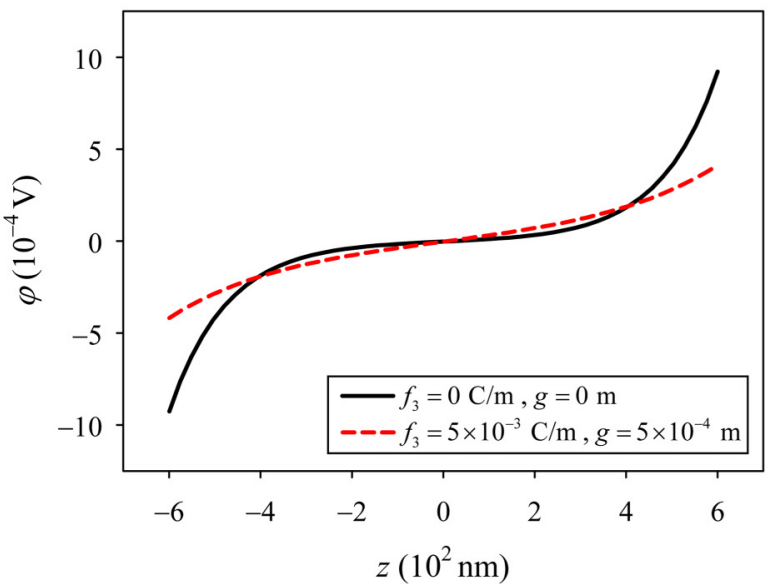

(c)

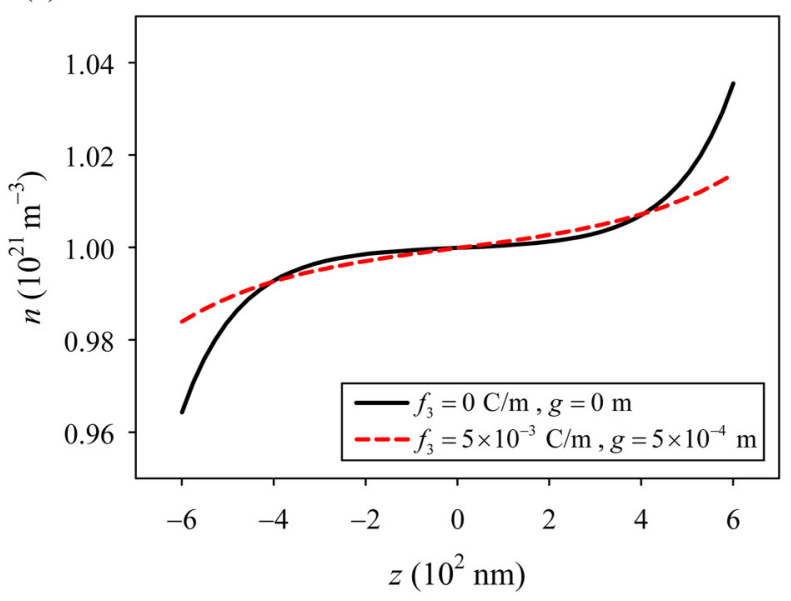

(b)

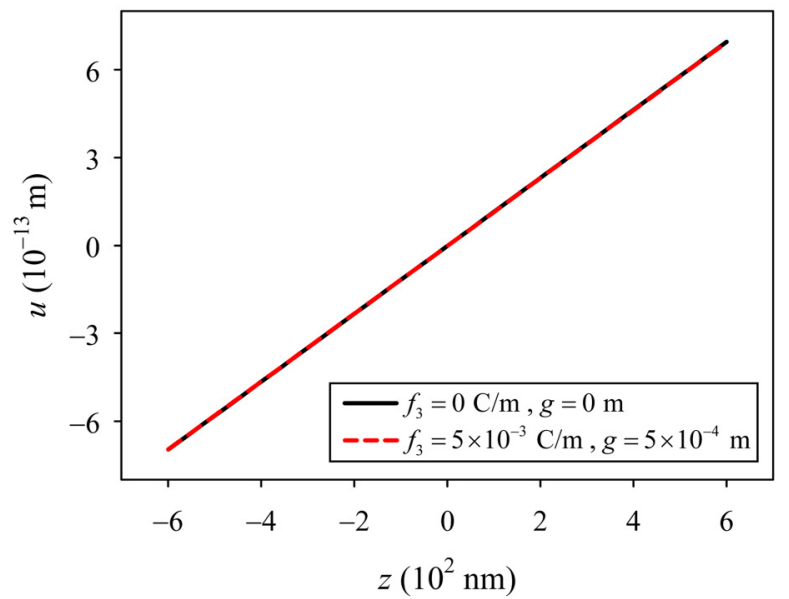

(d)

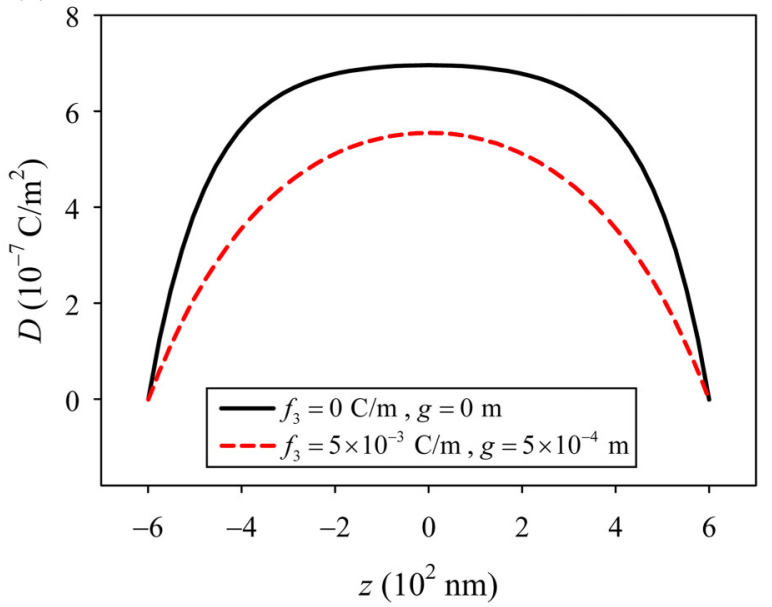

(e)

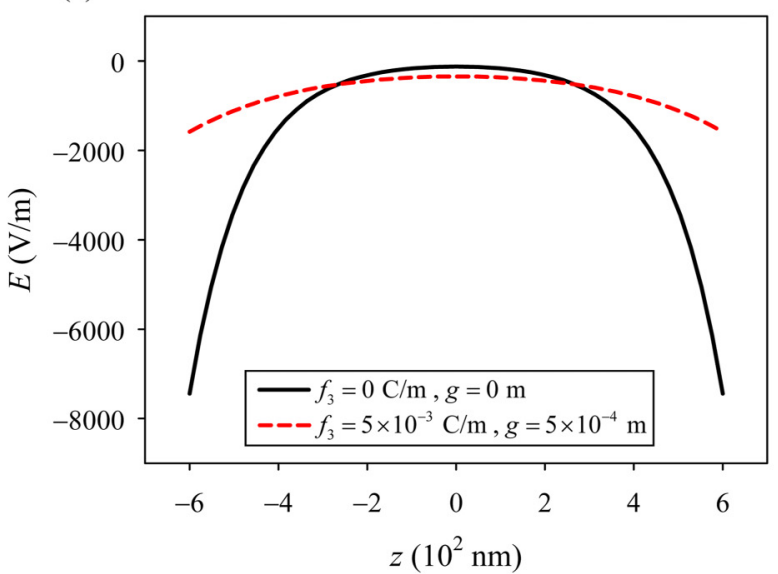

FIG. 2. Distributions along the nanowire with and without strain gradient and flexoelectric effects: (a) electric potential, (b) displacement, (c) carrier concentration, (d) electric displacement, and (e) electric field. 
By integrating Eq. (21b) twice, we have

$$
\begin{aligned}
u= & \frac{K_{1}}{r_{1}^{2}} \cosh r_{1} x+\frac{K_{2}}{r_{1}^{2}} \sinh r_{1} x+\frac{K_{3}}{r_{3}^{2}} \cosh r_{3} x+\frac{K_{4}}{r_{3}^{2}} \sinh r_{3} x \\
& +C_{5} x+C_{6},
\end{aligned}
$$

where $C_{5}$ and $C_{6}$ are integral constants.

Substituting $\varphi$ and $U$ into Eq. (15), the parameters $\left(K_{1}-K_{4}\right)$ can be obtained as

$$
K_{1}=\frac{e_{33} C_{1} r_{1}^{2}-f_{3} C_{2} r_{1}^{3}}{c_{33} g^{2} r_{1}^{2}-c_{33}},
$$

(a)

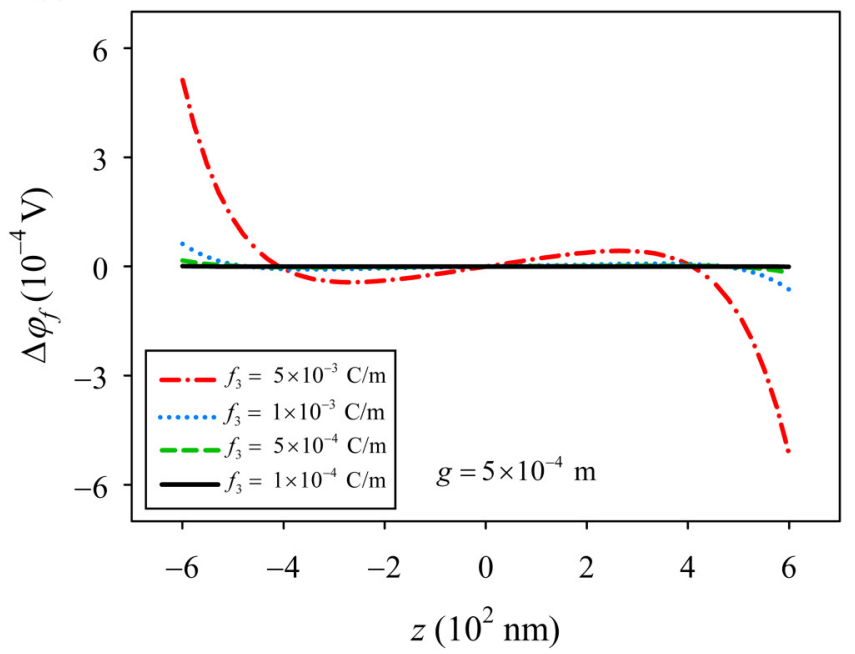

(c)

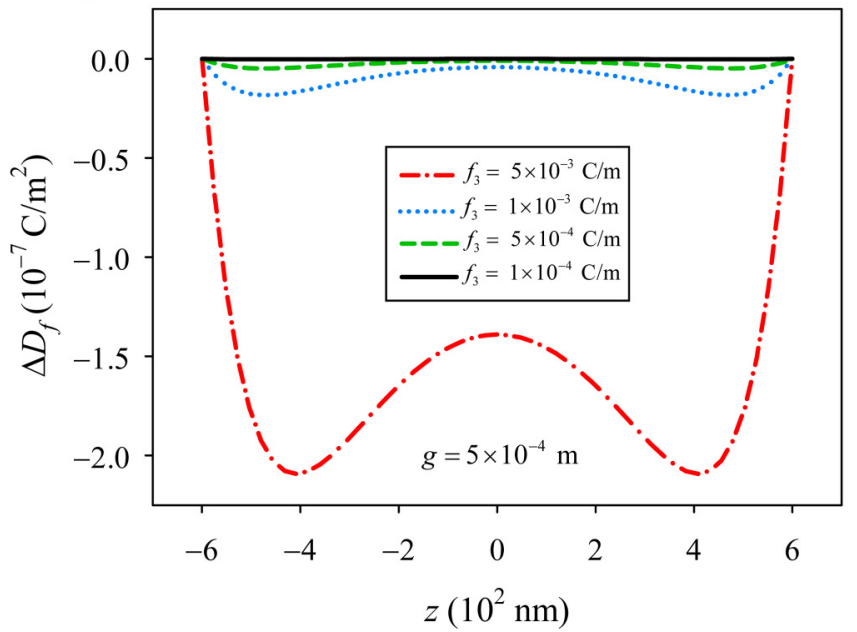

$$
\begin{aligned}
& K_{2}=\frac{e_{33} C_{2} r_{1}^{2}-f_{3} C_{1} r_{1}^{3}}{c_{33} g^{2} r_{1}^{2}-c_{33}}, \\
& K_{3}=\frac{e_{33} C_{3} r_{1}^{2}-f_{3} C_{4} r_{1}^{3}}{c_{33} g^{2} r_{3}^{2}-c_{33}}, \\
& K_{4}=\frac{e_{33} C_{4} r_{1}^{2}-f_{3} C_{3} r_{1}^{3}}{c_{33} g^{2} r_{3}^{2}-c_{33}} .
\end{aligned}
$$

Here, the independent parameters are $C_{1}-C_{6}$, and $K_{1}-K_{4}$ can be expressed in terms of $C_{1}-C_{4}$.

(b)

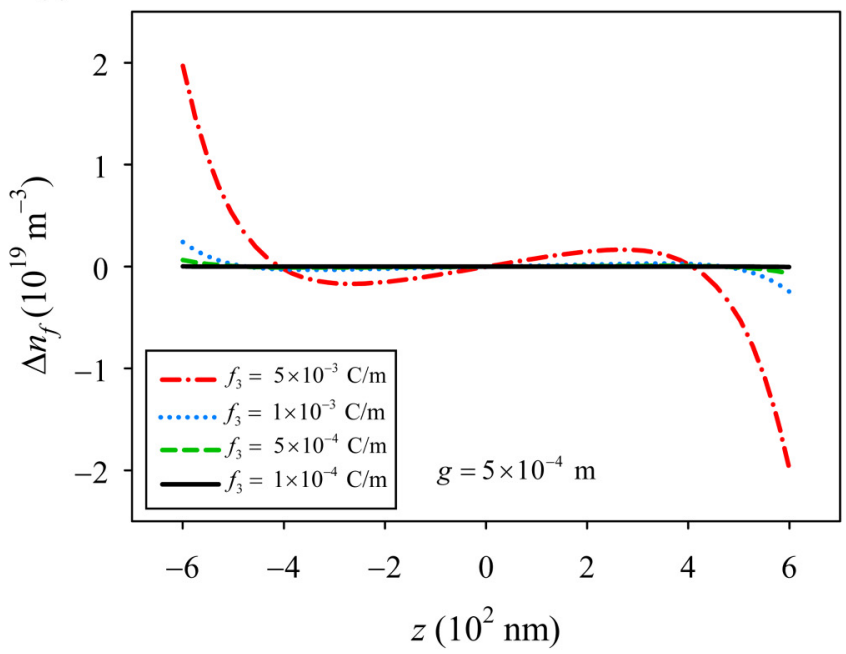

(d)

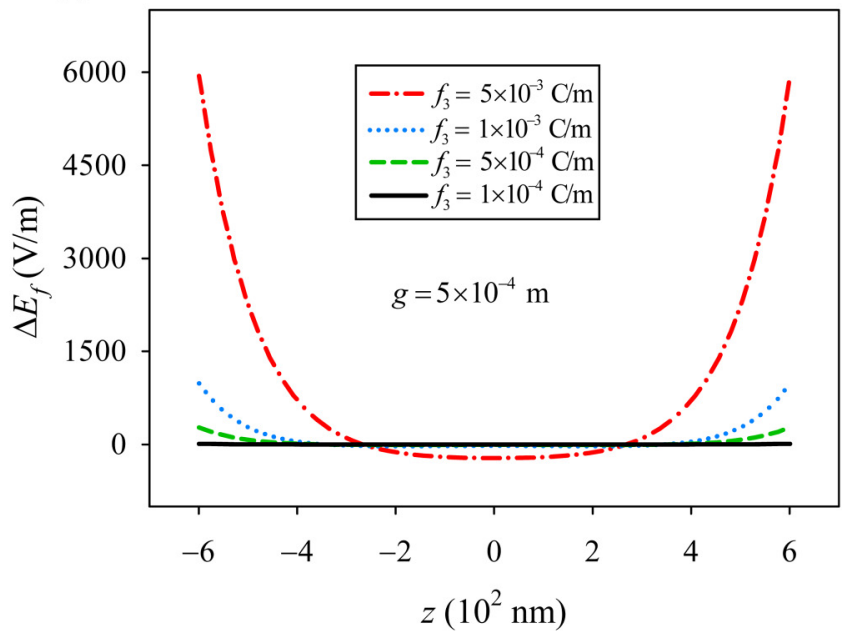

FIG. 3. Distributions along the nanowire with different flexoelectric coefficients: (a) electric potential, (b) carrier concentration, (c) electric displacement, and (d) electric field. 
Substituting Eqs. (3a) and (3b) into the expression for $\hat{\sigma}$ leads to

$$
\hat{\sigma}=c_{33} \frac{d u}{d z}+e_{33} \frac{d \varphi}{d z}-c_{33} g^{2} \frac{d^{3} u}{d z^{3}}-f_{3} \frac{d^{2} \varphi}{d z^{2}} .
$$

Then, by substituting $u$ and $\boldsymbol{\varphi}$, as well as Eqs. (23a)-(23d) into Eq. (24), we have

$$
\hat{\sigma}=C_{5} c_{33} .
$$

Finally, based on Eq. (10a), we can obtain

$$
C_{5}=\frac{\hat{\sigma}}{c_{33}}=\frac{F}{A c_{33}} .
$$

(a)

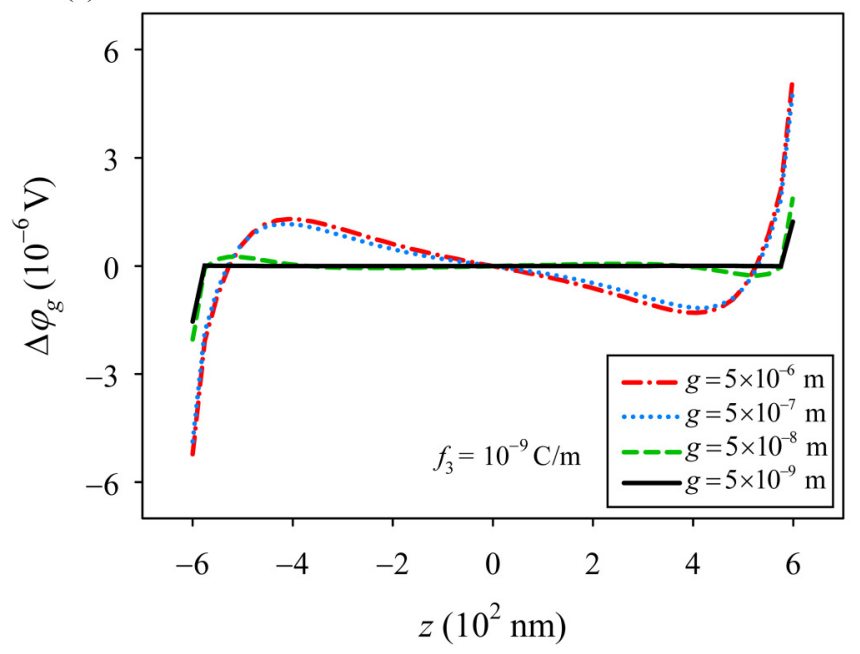

(c)

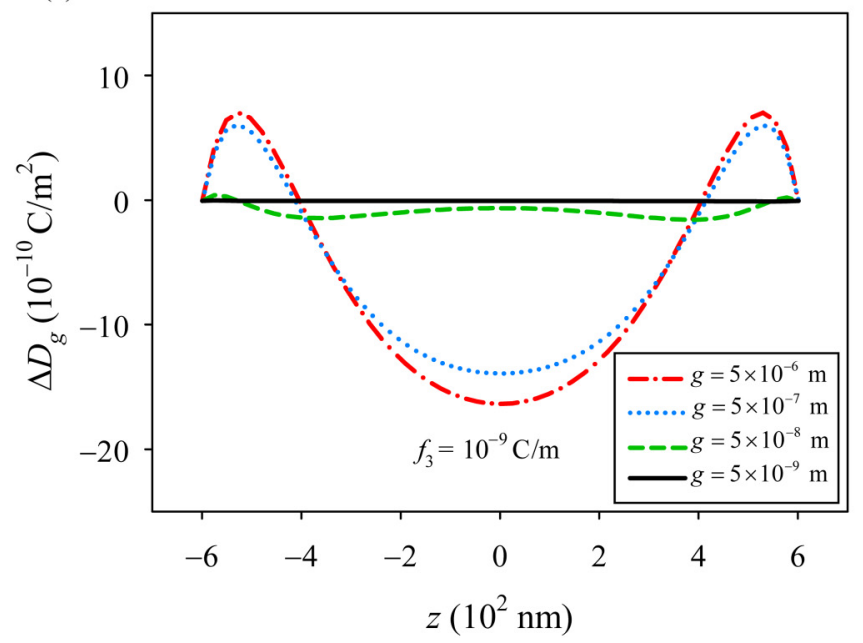

Similarly, substituting Eqs. (21) and (22) into the boundary conditions [i.e., Eqs. (10b) and (10c)] and using $u(0)=0$, the other five constants $\left(C_{1}-C_{4}\right.$ and $\left.C_{6}\right)$ can be obtained by solving linear algebraic equations.

\section{NUMERICAL RESULTS AND DISCUSSION}

Let us consider an $n$-type gallium nitride $(\mathrm{GaN})$ nanowire (see Fig. 1), with $2 L_{0}=1.2 \mu \mathrm{m}$ and $A_{0}=2.598 \times 10^{-14} \mathrm{~m}^{2}$ (radius $a=91 \mathrm{~nm}$ ). The applied force at the two ends is $F=8.5 \mathrm{nN}$, and the initial carrier concentration is $n_{0}=10^{21} \mathrm{~m}^{-3}$. The material parameters for $\mathrm{GaN}^{45}$ are $c_{33}=282.0 \mathrm{GPa}, e_{33}=0.61 \mathrm{C} / \mathrm{m}^{2}$, $k_{33}=9.38524 \times 10^{-11} \mathrm{C} / \mathrm{V} \mathrm{m}, \mu_{33}^{n}=0.0962 \mathrm{~m}^{2} / \mathrm{V} \mathrm{s}$, and $d_{33}^{n}=2.5$ $\times 10^{-11} \mathrm{~m}^{2} / \mathrm{s}$. It is worth noting that there is no available

(b)

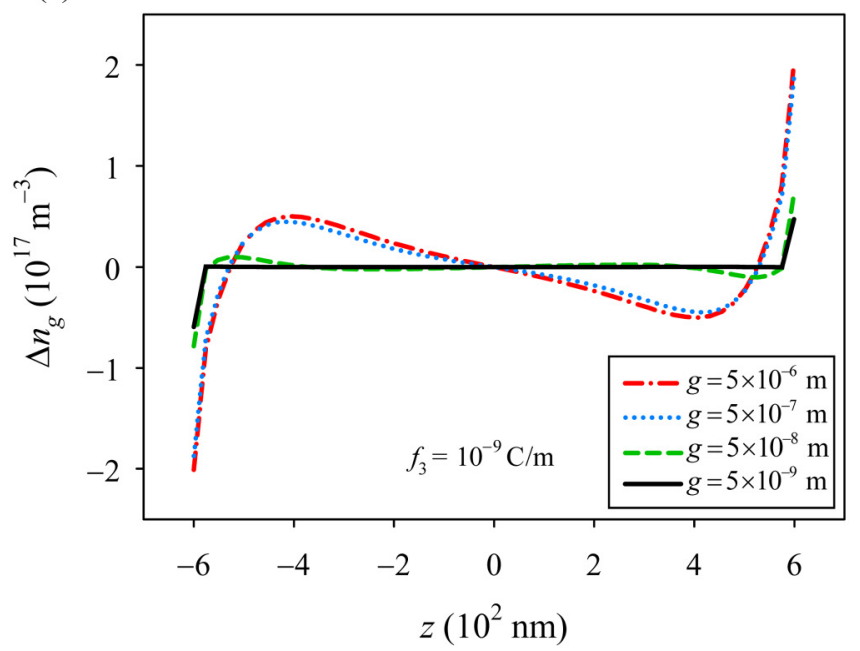

(d)

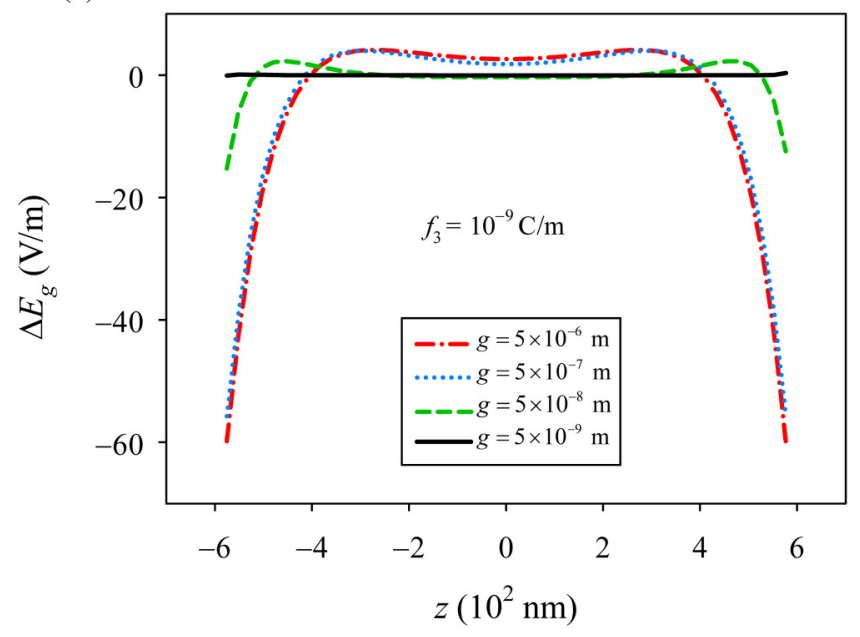

FIG. 4. Distributions along the nanowire with different non-local coefficients: (a) electric potential, (b) carrier concentration, (c) electric displacement, and (d) electric field in the case of $f_{3}=10^{-9} \mathrm{C} / \mathrm{m}$. 
flexoelectric coefficient for GaN. However, as reported in Refs. 35 and 46, the flexoelectric and non-local coefficients for ferroelectric ceramics are dependent on the temperature and fabrication methods, such as by a reduction reaction using graphite as the reducing agent or via a thermal treatment. According to experimental reports, the flexoelectric coefficient can reach the order of $10^{-3} \mathrm{C} / \mathrm{m} ;{ }^{46}$ hence, we choose flexoelectric coefficients in the range of $10^{-9}-10^{-3} \mathrm{C} / \mathrm{m}$.

To clarify the influence of the flexoelectric and strain gradient effects on various physical fields, relatively large flexoelectric and non-local coefficients were chosen. As shown in Fig. 2(a), the displacement was almost independent of the flexoelectricity and strain gradient. However, the flexoelectricity and strain gradients have an

(a)

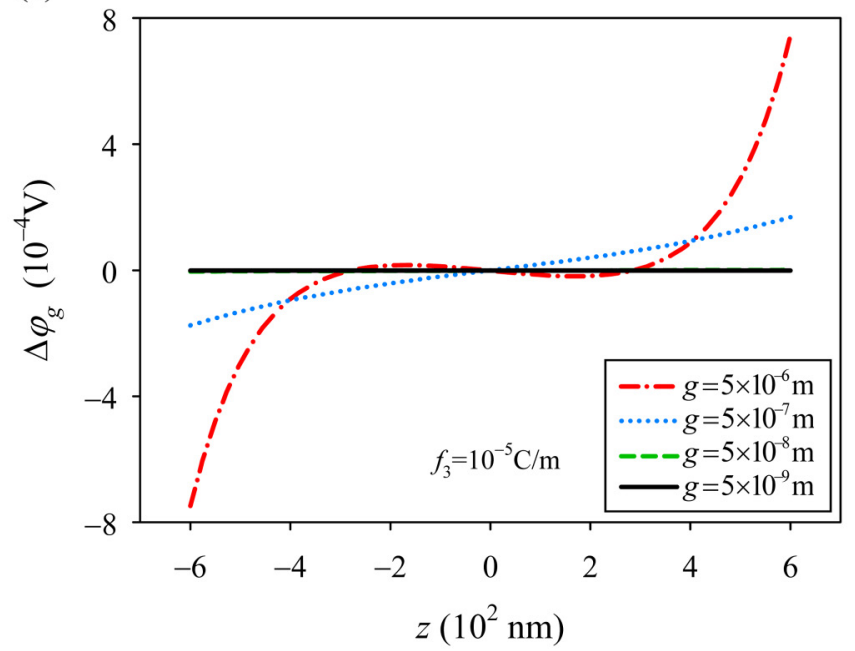

(c)

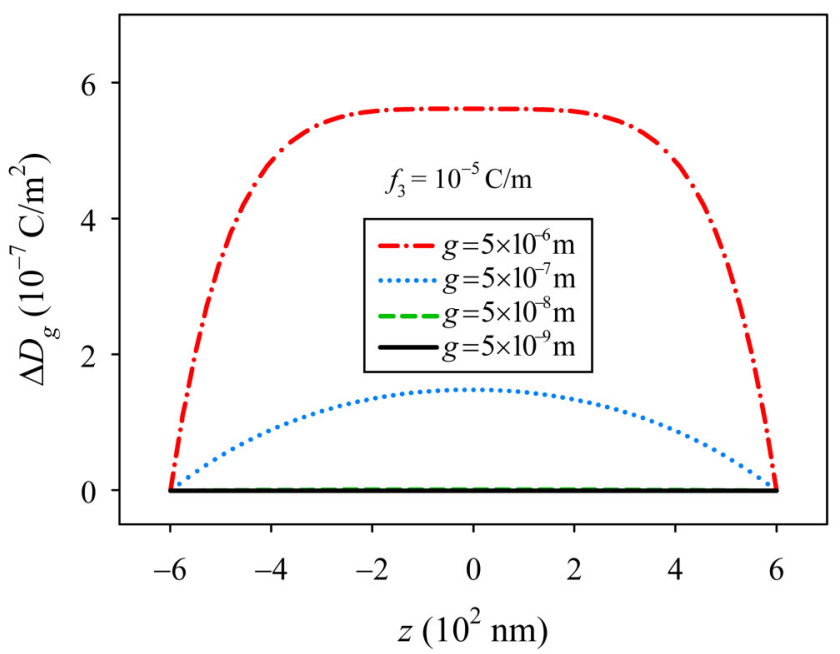

obvious influence on the potential, electron density, electric field, and electric displacement distributions.

Then, to study the influence of flexoelectricity alone, different flexoelectric coefficients were chosen and the other material parameters were held constant. To clearly show the influence of flexoelectricity, the distribution of a physical field was subtracted from the corresponding results without flexoelectricity (i.e., for the case of $f_{3}=0$, see Fig. 3). For example, $\Delta \varphi_{f}$ represents the electrical potential for the case of a certain nonzero flexoelectric coefficient minus that with a zero flexoelectric coefficient. As observed from Fig. 3, the flexoelectric effect has a large influence on the electric potential, carrier concentration, and electric field at both ends of the PSC nanowire and on the electric displacement at the middle. The influence

(b)

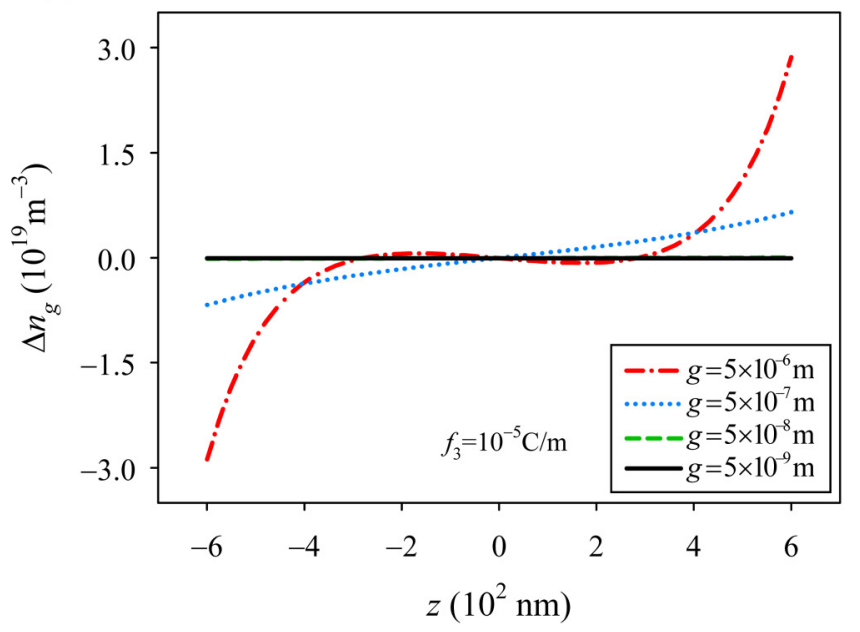

(d)

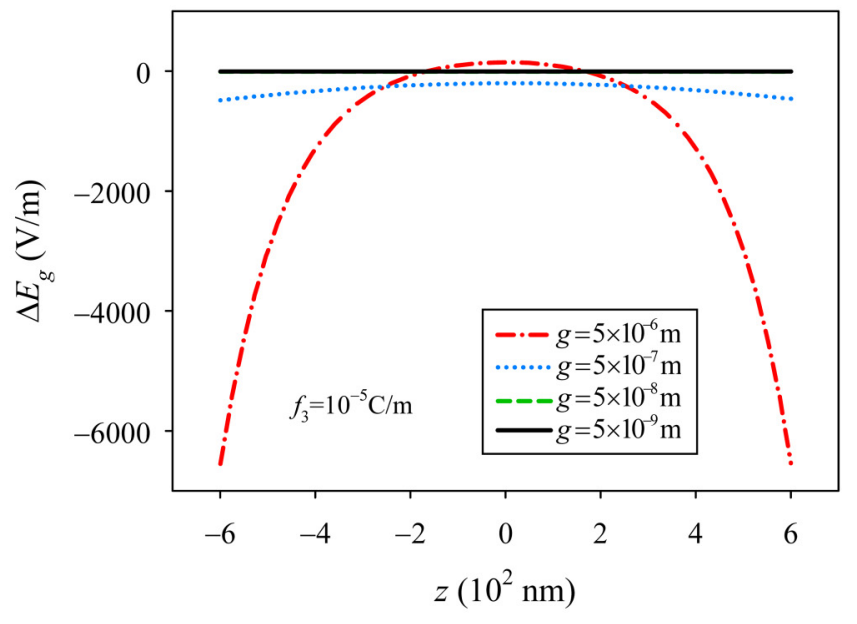

FIG. 5. Distributions along the nanowire with different non-local coefficients: (a) electric potential, (b) carrier concentration, (c) electric displacement, and (d) electric field in the case of $f_{3}=10^{-5} \mathrm{C} / \mathrm{m}$. 


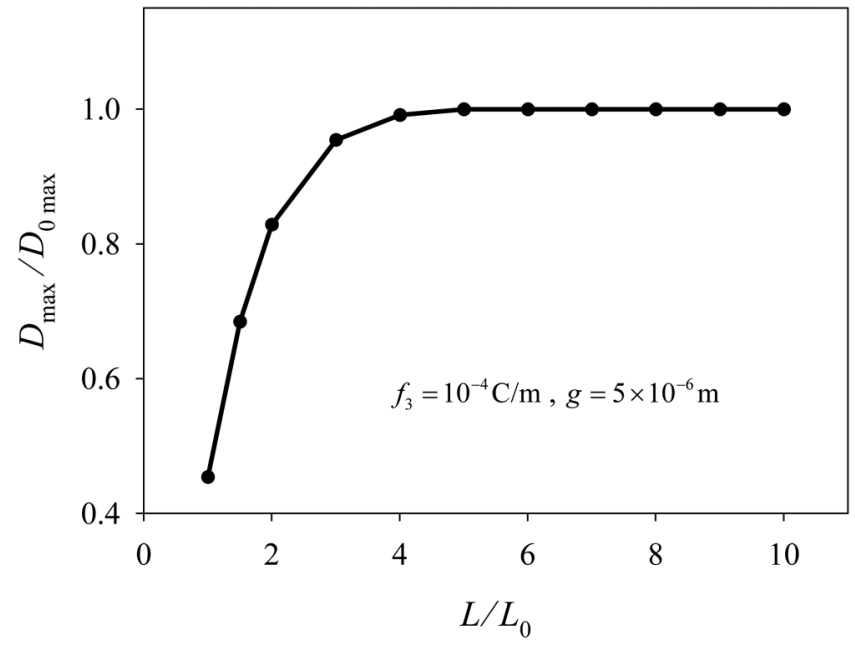

FIG. 6. The size effect of the PSC nanowire, where $D_{0 \max }$ is the maximum value of electric displacement in the case of $f_{3}=0$ and $g=0$.

increases as the flexoelectric coefficient increases. Furthermore, it can be seen in Figs. 2 and 3 that the electrical potential is positive at the right end, while $\Delta \varphi_{f}$ on the right end is negative. Therefore, the flexoelectric effect weakens the electric potential at the ends of the PSC nanowire.

To study the influence of the strain gradient, we selected different non-local coefficients and held the other material parameters constant. In a similar way, the result subtracted from that for the case of $g=0$ is defined as the influence of the strain gradient. As shown in Fig. 4, the strain gradient effect increases with increasing $g$. The strain gradient has a large influence on the electric potential, carrier concentration, and electric field of the PSC nanowire and on the electric displacement at the middle of the nanowire. In contrast, the strain gradient effect enhances the electric potential at the ends of the PSC nanowire (see Figs. 2 and 4). The inversion of $\Delta D_{g}$ toward the nanowire ends in Fig. 4 (c) is due to both $f_{3}$ and $g$.

Similarly, from Fig. 5, it can be observed that as $g$ increases, the influence of the strain gradient increases. However, on comparing Fig. 4 with Fig. 5, the magnitudes of $\Delta \varphi_{g}, \Delta n_{g}, \Delta D_{g}$, and $\Delta E_{g}$ for the case of $f_{3}=10^{-5} \mathrm{C} / \mathrm{m}$ are higher than for the case of $f_{3}=10^{-9} \mathrm{C} / \mathrm{m}$.

Finally, the maximum value of the electric displacement $D_{\max }$ (on the middle of the nanowire) is taken as an example to investigate the size effect. A similar geometrical configuration was applied with $F / A$ being a constant. It can be seen from Fig. 6 that when the length of a nanowire reduces to a critical value, $D_{\max } / D_{0 \max }$ decreases, where $D_{0 \max }$ is the maximum value of the electric displacement for the case of $f_{3}=0$ and $g=0$. A smaller length results in an increasingly significant size effect.

\section{CONCLUSION}

In summary, the governing equations and constitutive relationships for a 1D PSC nanowire were established with consideration of the flexoelectric and strain gradient effects. For the case of a small perturbation of the carrier concentration, theoretical solutions for a PSC nanowire can be obtained under an axial force. The analytical expressions were a combination of hyperbolic (or linear) functions and constants. The numerical results demonstrated that both the strain gradient and flexoelectricity have an influence on the electric potential distribution. In contrast with the weakening influence of flexoelectricity on the piezoelectricity of a PSC nanowire, the strain gradient effect results in an enhancement of the piezoelectricity, and its amplitude is mainly dependent on the flexoelectric and inner scale coefficients. Finally, it should be noted that our results were obtained for a nanowire under tension; for the case of a nanowire under a transverse force, the 1D beam model should be used.

\section{ACKNOWLEDGMENTS}

This work has been supported by the National Natural Science Foundation of China (NNSFC) (Nos. 11572289, 11702251, and 11702252).

\section{REFERENCES}

${ }^{1}$ J. S. Yang and H. G. Zhou, Int. J. Solids Struct. 42, 3171 (2005).

${ }^{2}$ G. J. Rees, Microelectron. J. 28, 957 (1997).

${ }^{3}$ R. S. Withers, IEEE Trans. Sonics Ultrason. 31, 117 (1984).

${ }^{4}$ A. R. Huston, Rev. Lett. 4, 505 (1960).

5Z. L. Wang, Adv. Mater. 24, 4632 (2012).

6. S. Yang, X. M. Yang, and J. A. Turner, Arch. Appl. Mech. 74, 288 (2004).

7J. S. Yang, Y. C. Song, and A. K. Soh, Arch. Appl. Mech. 76, 381 (2006).

${ }^{8}$ A. R. Huston and D. L. White, J. Appl. Phys. 33, 40 (1962).

${ }^{9}$ M. H. Zhao, S. J. Ma, C. S. Lu, C. Y. Fan, and G. S. Qin, Ceram. Int. 44, 12648 (2018).

${ }^{10}$ Z. L. Wang, Adv. Mater. 15, 432 (2003).

${ }^{11}$ Z. L. Wang, Nano Today. 5, 540 (2010).

${ }^{12}$ B. Kumar and S. W. Kim, J. Mater. Chem. 21, 18946 (2011).

${ }^{13}$ Z. L. Wang, Science 312, 242 (2006).

${ }^{14}$ P. X. Gao, J. Song, J. Liu, and Z. L. Wang, Adv. Mater. 19, 67 (2007).

${ }^{15}$ J. Song, J. Zhou, and Z. L. Wang, Nano Lett. 6, 1656 (2006).

${ }^{16}$ J. H. He, C. L. Hsin, J. Liu, L. J. Chen, and Z. L. Wang, Adv. Mater. 19, 781 (2007).

${ }^{17}$ C. S. Lao, Q. Kuang, Z. L. Wang, M. C. Park, and Y. Deng, Appl. Phys. Lett. 90, 262107 (2007).

${ }^{18}$ A. Erturk and D. J. Inman, Smart Mater. Struct. 17, 065016 (2008).

${ }^{19}$ Y. Gao and Z. L. Wang, Nano Lett. 7, 2499 (2007).

${ }^{20}$ G. Mantini, Y. Gao, A. D'Amico, C. Falconi, and Z. L. Wang, Nano Res. 2, 624 (2009)

${ }^{21}$ Y. Gao and Z. L. Wang, Nano Lett. 9, 1103 (2009).

${ }^{22}$ C. L. Zhang, X. Y. Wang, W. Q. Chen, and J. S. Yang, Smart Mater. Struct. 26, 025030 (2017)

${ }^{23}$ X. Y. Dai, F. Zhu, Z. H. Qian, and J. S. Yang, Nano Energy. 43, 22 (2018).

${ }^{24}$ R. R. Cheng, C. L. Zhang, W. Q. Chen, and J. S. Yang, J. Appl. Phys. 124, 064506 (2018).

${ }^{25}$ G. L. Wang, J. X. Liu, X. L. Liu, W. J. Feng, and J. S. Yang, J. Appl. Phys. 124, 094502 (2018).

${ }^{26}$ W. L. Yang, Y. T. Hu, and J. S. Yang, Mater. Res. Express 6, 025902 (2019).

${ }^{27}$ G. Yang, J. Du, J. Wang, and J. S. Yang, Acta Mech. 229, 4663 (2018).

${ }^{28}$ N. A. Fleck, G. M. Muller, M. F. Ashby, and J. W. Hutchinson, Acta Metall. Mater. 42, 475 (1994).

${ }^{29}$ D. C. C. Lam, F. Yang, A. C. M. Chong, J. Wang, and P. Tong, J. Mech. Phys. Solids 51, 1477 (2003).

${ }^{30}$ M. Kandaz and H. Dal, Arch. Appl. Mech. 88, 2051 (2018). 
${ }^{31}$ E. C. Aifantis, Int. J. Eng. Sci. 30, 1279 (1992).

${ }^{32}$ B. C. Altan and E. C. Aifantis, J. Mech. Behav. Mater. 8, 231 (1997).

${ }^{33}$ J. E. Spanier, A. M. Kolpak, J. J. Urban, I. Grinberg, L. Ouyang, W. S. Yun, A. M. Rappe, and H. Park, Nano Lett. 6, 735 (2006).

${ }^{34}$ W. J. Yang, T. T. Hu, X. Liang, and S. P. Shen, Arch. Appl. Mech. 88, 629 (2018).

${ }^{35}$ L. Cross, J. Mater. Sci. 41, 53 (2006).

${ }^{36}$ J. Hong and D. Fang, Appl. Phys. Lett. 92, 012906 (2008).

${ }^{37}$ J. Hong and D. Fang, J. Appl. Phys. 104, 064118 (2008).

${ }^{38}$ Y. Zhang, J. Hong, B. Liu, and D. Fang, Nanotechnology 20, 405703 (2009).
${ }^{39}$ M. Majdoub, P. Sharma, and T. Cagin, Phys. Rev. B 77, 125424 (2008).

${ }^{40}$ A. Askar, P. C. Y. Lee, and A. S. Cakmak, Phys. Rev. B 1, 3525 (1970).

${ }^{41}$ C. Liu, S. Hu, and S. P. Shen, Smart Mater. Struct. 21, 115024 (2012).

${ }^{42}$ X. Liang, S. Hu, and S. P. Shen, Smart Mater. Struct. 26, 035050 (2017).

${ }^{43} \mathrm{~K}$. Wang and B. Wang, Nanotechnology 29, 255405 (2018).

${ }^{44}$ D. L. White, J. Appl. Phys. 33, 2547 (1962).

${ }^{45}$ G. S. Qin, S. J. Ma, C. S. Lu, G. Wang, and M. H. Zhao, Ceram. Int. 44, 4169 (2018).

${ }^{46}$ W. Zhou, P. Chen, and B. Chu, IET Nanodielectr. 2, 83 (2019). 